Unsere Versuche in dieser Hinsicht umfaßten eine Vakz.inationstherapie mit einer alls dem Proteusstamm $\mathrm{X}_{19}$ hergestellten Vakzine.

Die Herstellung der Vakzine war dir allgemein übliche:

Abschwemmungen ciner 24 Stunden alten Schrägagarreinkultur wurden nach fünf Minuten langem Schütteln eine halbe Stunde bci $60^{\circ}$ abgetötet und mit $0,5 \mathrm{ecm} 5 \%$ igcr Karbolsäurelösung versetat

Die Therapic wurde mit $0,05 \mathrm{ccm}$ subkutan begonnen. Nach zwei Tagen $0,1 \mathrm{ccm}$, nach weiteren zwei Tagen $0,2 \mathrm{ccm}$ intravenös (am Vormittag). Während bei subkutaner Verabfolgung keinerlei Effekt erzielt wurde, kam es nach intravenöser $\Lambda$ nwendung $\mathrm{zu}$ einem ausgesprochenen anaphylaktischen Symptomenbild mit Atemnot, weiterer Fiebersteigerung, später auch Durchfällen. Calcium chloratum (als Ringerlösung), Kampfer und Adrenalin halfen diese Reaktionen überwinden, das Fieber sank, und die Temperatur blicb normal. Es empfiehlt sich, die Vakzination sofort intravenös mit kleinen Dosen $(0,05-0,1$ ccmı) zu beginnen, je nach den Kräftezustand des Kranken richtet sich die Dosierung. Zuvor und während der Reaktion muß man reichlich Herzmittel sowie Calcium chloratum (als Lösung 10,0: 200,0 am Tage zuvor zweimal ein Eßlöffel, ebenso am gleichen Tage), verabfolgen, damit eine stürmische Reaktion leichter überwunden wird. Bei dekrepiden Kranken mit verändertem Herzgefäßsystem ist die Vakzinierung zil widerraten.

Auch wenn man annimmt, daß es sich um eine lialbspezifische Vakzinierung liandelt, wäre die Nachprüfung zu empfehlen. Daß die Anregung von Immunkörperbildung gegen Proteus $\mathrm{X}_{19}$ bei Fleckfieber konstant und deshalb wohl auch bedeutungsvoll ist, liegt durchaus nahe. Zudem war der von

\section{Behandlung und Schutzimpfung bei Fleckfieber mittels Vakzinierung mit Proteus $X_{19}$.}

\author{
Von Prof. Dr. R. Hilgermann,
}

Jirektor des Kgl. Instituts für Hygiene und Infektionskrankheiten in Saarbriuken,

und Dr. W. Arnoldi,

Assistent der II. Mel. Klinik der Kgl. Charité, Berlin, und Stabsarz.t d. R.

Durch die Forschungen von Prowazek und RochaLima war die Kenntnis über den Erreger des Fleckfiebers bedeutend gefördert worden. Trotz der für die Erregernatur der Rickettsia Prowazeki überzeugend sprechenden Infektionsversuche mit Läusen blieb das spezifische Agglutinationsphänomen mit dem Weilschen Proteusstamm $X_{19}$ ungeklärt. An einer großen Anzahl in der Hygienischen Untersuchungsstelle des Generalgouvernements Warschau untersuchter Blutproben von Fleekfieberkranken konnten wir uns von der Spezifizität der Reaktion überzeugen. Für letzteres sprach auch besonders das eharakteristische Ansteigen des Titers im Verlaufe der Erkrankung, sein Absinken gegen Ende derselben bzw. in der Rekonvaleszenz. Bei einer großen Anzahl von Untersuchungen mit von anderen Erkrankungen stammenden Sera wurde Mitagghntination nur in verschwindender Zahl, und zwar höchstens in der Verdünuung 1:50, festgestellt. Zeigten Blutproben bei verdächtigen, aber klinisch nicht sicherem Krankheitsbilde einmal höhere Agglutinationswerte, so stammten diese ans der Ungebung Fleckfieberkranker.

Nach unserer gesamten Kenntnis von dem Wesen der Agglutination muß dieser allgemein festgestellten Spezifizität der Agglutination bei Fleckfieberkranken unbedingt eine gewisse Bedeutung in bezug auf eine eventuelle Mitbeteiligung des Protensstammes $\mathrm{X}_{19}$ für das Zustandekommen der Infektion beigemessen werden.

Die Dienes') gelnngene Züchtung der Weilschen Bakterien aus dem Blute Fleckfieberkranker spricht weiterhin für letztere Annahme.

Selbst wenn dieser Proteusstamm als direkter Erreger vollkommen abzulehnen wäre, kommt ihm vielleicht zum mindesten eine gewisse Bedeutung in Symbiose mit einer anderen Bakterien- oder Chlamydozoenart zu.

Von letzteren Ueberlegungen ausgehend, war vielleicht damit auch ein Weg zu therapeutischen Maßnahmen gewiesen. mus erzielte Erfolg, wie bereits hervorgehoben, augenscheinlich: typischer Temperaturabfall und Genesung bei anfänglicher großer Schwäche.

Naclistehende Temperatırkurve zeigt den durch die Vak. zination erzielten Erfolg.

Es wurde weiter eine Reilhe von Männern und Frauen, die wegen Anstecknngsverdacht isoliert worden waren, mit der Vakzine einmalig 0,1 oder zwei. malig 0,1 und 0,2 subkutan schutzgeimpft. Ohne besondere örtliclie oder allgemeine Beschwerden, bis auf die üblichen Impferscheinungen, gelang es schnell und leicht, hohen Agglutinationstiter zu erzeugen. Ein Urteil über den Erfolg bzw. den Wert der Schutzinıpfung ließe sich jedoch erst nach Beobachtung an einem großen Material erbringen.

Leider mußten die Versuche infolge Versetzung abgebrochen werden. Immerhin scheint es uns doch wichtig genug, auf sie behufs Nachprüfıng hinzuweisen. Denn jeder therapeutische Versuch, welcher geeignet wïre, der Fleckfiebererkrankung ilıren bösartigen Charakter zu nehmen, würde einen Fortscliritt bedeuten. 